\section{Bruises around the umbilicus in an infant}

\author{
Sam Hassan, Ghassan Nakib, \\ Mary J. Saviour \\ Departments of Pediatrics and Neonates, \\ Mediclinic City Hospital, Dubai Health \\ Care City, Dubai, United Arab Emirates
}

\begin{abstract}
The most common causes of umbilical discharge in infancy are infection and umbilical granuloma that may be treated by antibiotics and topical application of silver nitrate subsequently. If the umbilical discharge persists or if there is any abnormal discoloration around the umbilicus, it is important to investigate for underlying congenital abnormality that may be cured by surgical intervention. Unusual presentation of omphalomesenteric duct cyst has been reported in literature. We report, for the first time as far as our search is concerned, a case of a 16-month-old infant who presented initially with persistent umbilical discharge and finally with bruising around the umbilicus in keeping with Cullen's sign. A diagnosis of omphalomesenteric duct cyst containing pancreatic tissue was made on histopathological examination. This case emphasizes that, a persistent umbilical discharge and or discoloration around the umbilicus should be further investigated and an omphalomesenteric duct cyst can present as Cullen's sign.
\end{abstract}

\section{Introduction}

Omphalomesenteric duct is an embryonic communication between midgut and yolk sac. It typically involutes between 9-18 weeks of gestation. The omphalomesenteric duct cyst is an embryologic remnant of the yolk stalk. If the yolk stalk does not obliterate completely, various portions may persist giving rise to entities like Meckel's Diverticulum, intra-abdominal cyst, enteric fistula to the umbilicus or a cyst or a polypoid umbilical cord mass including an omphalomesenteric cyst. ${ }^{1}$ The cyst is lined by columnar mucin secreting epithelium. Eventually this cyst may contain gastric, colonic, pancreatic or small intestinal epithelium. ${ }^{1}$ Cullen's sign is described as bruising with superficial edema in the subcutaneous fatty tissue around the periumbilical region and is also known as periumbilical ecchymosis. ${ }^{2}$ This sign can predict acute pancreatitis. We report for the first time a case of omphalomesenteric duct (OMD) cist present- ed as Cullen's sign due to inflammation of the ectopic pancreatic tissue.

\section{Case Report}

A 16-month-old male infant presented to our outpatient department, many times with problems related to umbilicus. First seen at 2 months of age in the well-baby clinic with history of persistent oozing umbilicus after birth, which resolved after neosporin ointment. The next visit was at 3 months of age with inflamed erythematous oozing umbilicus diagnosed as candidiasis/intertrigo and treated with antifungal cream. An umbilical swab culture showed heavy growth of streptococcus agalactciae and treated with a course of amoxicillin. In spite of the antibiotic treatment erythematous umbilical rash persisted with clear fluid oozing. A repeated culture showed the same bacteria. An ultra sound was done which showed evidence of mixed echogenic space occupying lesion $1.8 \times 1.4 \mathrm{~cm}$ anterior superior to the dome of bladder with small trickle of Doppler signal within the lesion and appearance suggestive of inflammatory lesion extending from umbilicus towards the dome of bladder. Further evaluation by computed tomography scan was advised.

There after the baby lost follow up. At 16 months of age the child presented to Out Patients Department with faint bruises around the umbilicus. There was no history of fever, vomiting or excessive crying. On examination baby was hemodynamically stable and there was bruises around the subcutaneous tissue in the anterior abdominal wall over the umbilicus in keeping with Cullen's sign (Figure 1).

An ultrasound showed a well-defined echo indicating a cystic lesion at the site of umbilicus extending to both sides of midline and measuring $3 \times 2.5 \times 1.1 \mathrm{~cm}$ with a solid vascular component seen along the lateral portion measuring $10 \times 6 \mathrm{~mm}$, which was also partially connected to the overlying umbilicus and no definite tract seen with bladder with a conclusion of an omphalomesenteric cyst or infected urachal cyst and the child was referred to pediatric surgeon and surgical excision done.

On excision the mass was firm and no clear margin identified due to the chronic inflammatory process, and the mass was extraperitoneal extending directly under the umbilicus. This was totally excised and specimen sent to pathology (Figure 2).

The histopathology showed sections of fibro adipose tissue with few inflammatory cells and lobes of normal exocrine pancreatic tissue with ducts (Figure 3 ).

Post-operative period was uneventful, discharged from hospital. On follow up baby was healthy and no bruises were found around the umbilicus.
Correspondence: Sam Hassan, Departments of Pediatrics \& Neonates Mediclinic City Hospital, Dubai Health Care City, P.0. Box 505004, United Arab Emirates.

Tel. +971.4.4359999. Fax: +971.4.4359900.

E-mail: sam.hassan@mediclinic.ae

Key words: Cullens sign; umbilical bruises; omphalomesenteric duct cyst; pancreatitis.

Acknowledgements: the authors wish acknowledge the cooperation and support from the child's mother referred in this report and staff of Mediclinic City Hospital, Dubai.

Contributions: the authors contributed equally.

Conflict of interest: the authors declare no potential conflict of interest.

Received for publication: 22 April 2016.

Revision received: 10 June 2016.

Accepted for publication: 10 June 2016.

This work is licensed under a Creative Commons Attribution NonCommercial 4.0 License (CC BYNC 4.0).

(C) Copyright S. Hassan et al., 2016

Licensee PAGEPress, Italy

Pediatric Reports 2016; 8:6555

doi:10.4081/pr.2016.6555

\section{Discussion}

Omphalomesenteric duct cyst remnants are congenital anomalies associated with primitive yolk sac which persists in approximately $2 \%$ of population with higher incidence in male 2:1 and the most common to be Meckel's diverticulum. A study of 217 children with OMD remnants revealed that $40 \%$ can be symptomatic like umbilical hernia, umbilical discharge, intestinal obstruction, rectal bleeding or abdominal pain and these symptoms appear to be age dependent and approximately $80 \%$ of these present before the age of two. ${ }^{3}$

An extensive review of the medical literature has brought to our notice only 3 cases of umbilical cysts, six year old presenting as umbilical mass, ${ }^{4}$ two year old girl presenting as umbilical nodule,, 5 and six month old baby presenting as persistent umbilical discharge. ${ }^{6}$

Omphalomesenteric duct cyst may contain ectopic tissue and the most commonly found are gastric or pancreatic tissue.

Cullen's sign is described as superficial edema with bruising in the subcutaneous fatty tissue around the periumbilical region as a result of inflammation of pancreas.

Our case was an omphalomesenteric duct 
cyst containing pancreatic tissue, which presented as Cullen's sign. To the best of our knowledge the clinical finding cited, omphalomesenteric duct cyst presenting as Cullen's sign is the first of its kind being published.

Cullen's sign was first described by Thomas Cullen in 1918, classically associated with hemorrhagic pancreatitis but can also be associated with other conditions.

The Conditions associated with Cullen's sign are: i) pancreatitis; ii) ruptured ectopic pregnancy; iii) ruptured spleen; iv) ruptured aortic aneurysm; v) ruptured common bile duct; vi) perforated duodenal ulcer; vii) hepatocellular carcinoma; viii) hepatic lymphoma; ix) metastatic thyroid carcinoma; $x$ ) percutaneous liver biopsy.

Cullen's sign may coexist with grey Turner's sign, bruising on lateral aspect of abdominal wall or flanks. ${ }^{7}$ Periumbilical ecchymosis is due to the action of pancreatic enzymes react-

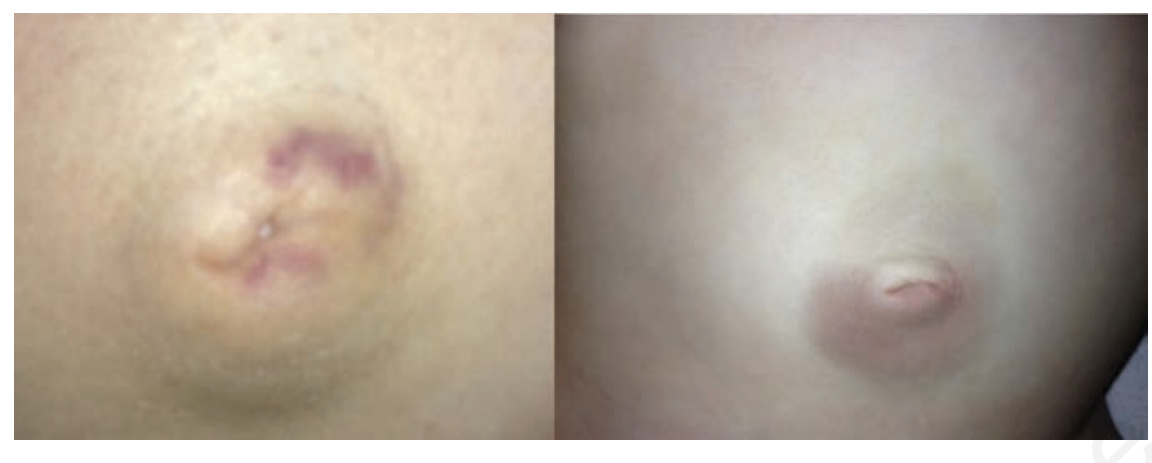

Figure 1. Umbilicus with bruises.

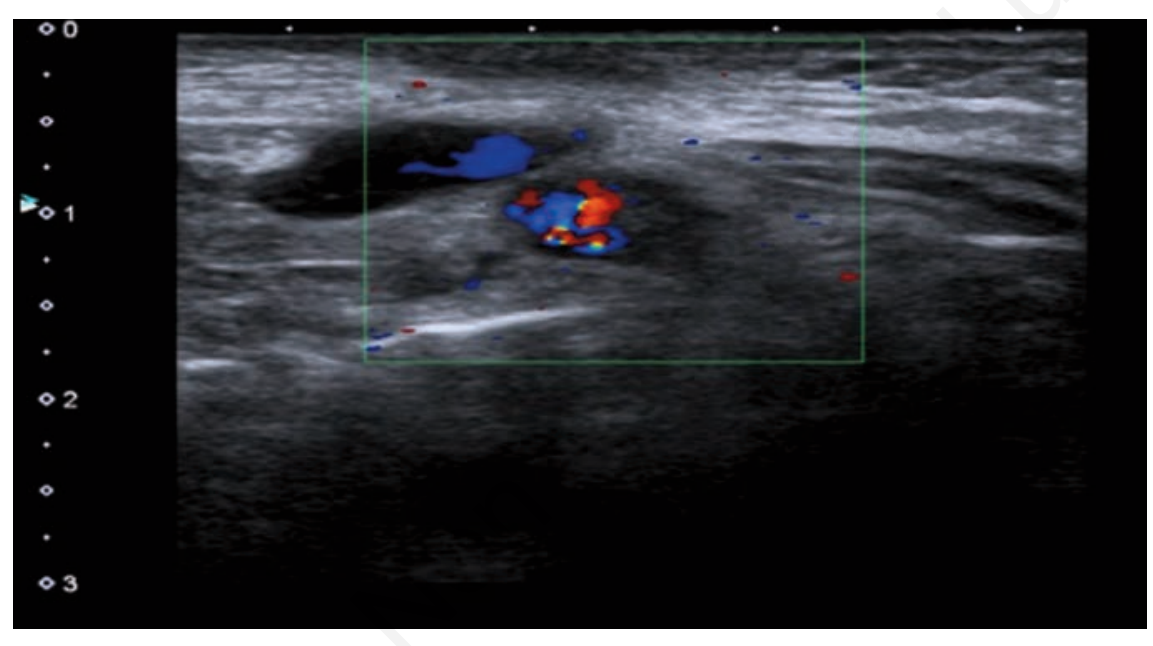

Figure 2. Cystic lesion under umbilicus with solid vascular component.

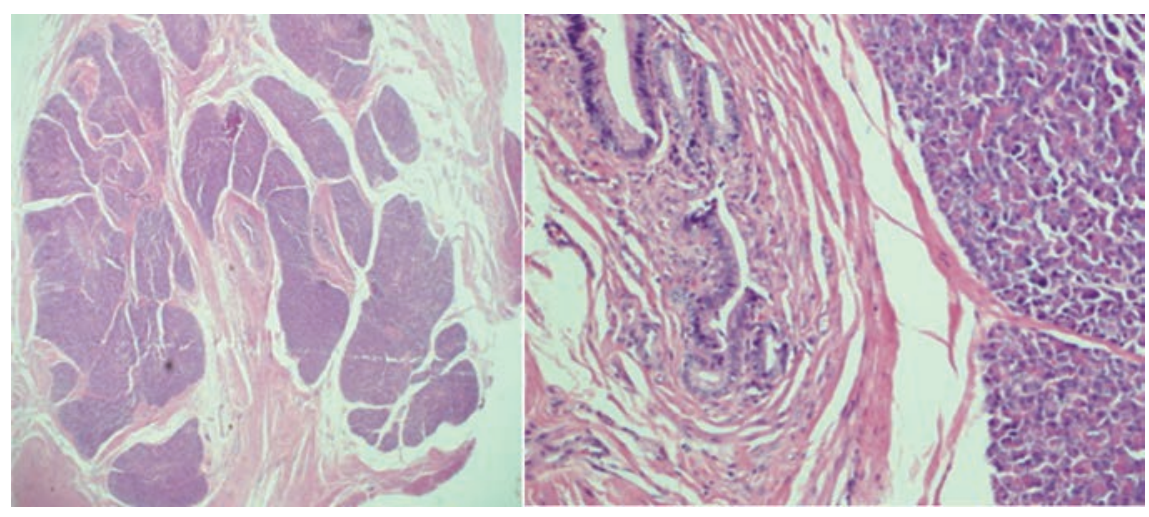

Figure 3. Histopathology showing exocrine pancreatic tissue with ducts. ing with adipose tissue in the abdomen resulting in diffusion of blood along the periumbilical tissue producing the discoloration around the navel, but this has not been widely accepted as periumbilical ecchymosis can be there in the absence of pancreatitis. Diffusion along the falciform ligament can also produce this. Discoloration is typically blue or purple but can be green or yellow depending on the stage of erythrocyte breakdown. ${ }^{7}$

The differential diagnosis of periumbilical erythema/bleeding includes: i) periumbilical cellulitis; ii) Sister Mary Joseph's sign a metastatic spread of an intra-abdominal malignancy to the umbilicus; iii) subcutaneous administration of heparin; iv) psoriasis may develop as umbilical erythema with shiny scales; v) umbilical endometriosis; vi) rarely adenocarcinoma of urachal remnants.

In our case we haven't done serum amylase level, which might be increased in acute inflammation of the pancreatic tissue.

\section{Conclusions}

Omphalomesenteric cyst duct can present as Cullen's sign. Early recognition of the sign, relevant investigation and appropriate referral to surgeon may be warranted.

\section{References}

1. Mccalla C0, Lajinian S, Desouza D, et al. Natural history of antenatal omphalomesenteric duct cyst. J Ultrasound Med 1995; 14:639-40.

2. Rahbour G, Ullah MR, Yassin N, Thomas GP. Cullen's sign - Case report with a review of the literature. Int J Surg Case Rep 2012;3:143-6.

3. Vane DW, West KW, Grosfeld JL. Vitelline duct anomalies. Experience with 217 childhood cases. Arch Surg 1987;122:542-7.

4. Iwasaki M, Taira K, Kobayashi H, Saiga T. Umbilical cyst containing ectopic gastric mucosa originating from an omphalomesenteric duct remnant. J Pediatr Surg 2009;44:2399-401.

5. Ballester I, Betlloch I, Pérez-Crespo M, et al. Atypical presentation of an omphalomesenteric duct cyst. Dermatol Online J 2009; 15:13.

6. Tamilselvan K, Mohan A, Cheslyn-Curtis S, Eisenhut M. Persistent umbilical discharge from an omphalomesenteric duct cyst containing gastric mucosa. Case Rep Pediatr 2012;2012:482185.

7. Marinella MA. Cullen's sign. Hosp Physician 1999;35:35-6. 